\title{
Cerebrofacial vascular metameric syndrome is caused by somatic pathogenic variants in PIK3CA
}

\author{
Sarah E. Sheppard, ${ }^{1,2}$ Victoria R. Sanders, ${ }^{1,2}$ Abhay Srinivasan, ${ }^{2,3}$ Laura S. Finn, ${ }^{4}$ \\ Denise Adams, ${ }^{2,5}$ Andrew Elton, ${ }^{6}$ Catherine Amlie-Lefond, ${ }^{7}$ Zoe Nelson, ${ }^{8}$ \\ Victoria Dmyterko, ${ }^{9}$ Dana Jensen, ${ }^{9}$ Kaitlyn Zenner, ${ }^{10,11}$ Jonathan Perkins, $^{10,11}$ \\ and James T. Bennett $8,9,11$
}

\begin{abstract}
${ }^{1}$ Division of Human Genetics, Department of Pediatrics, ${ }^{2}$ Comprehensive Vascular Anomaly Program, ${ }^{3}$ Division of Interventional Radiology, Department of Radiology, ${ }^{4}$ Department of Pathology, ${ }^{5}$ Division of Oncology, Department of Pediatrics, Children's Hospital of Philadelphia, Philadelphia, Pennsylvania 19104, USA; ${ }^{6}$ University of Minnesota School of Medicine, Minneapolis, Minnesota 55455, USA; ${ }^{7}$ Department of Neurology, Seattle Children's Hospital, Washington 98105, USA; ${ }^{8}$ Seattle Children's Hospital, Division of Genetic Medicine, Department of Pediatrics, University of Washington, Seattle, Washington 98105, USA; ${ }^{9}$ Center for Developmental Biology and Regenerative Medicine, Seattle Children's Research Institute, Seattle, Washington 98101, USA; ${ }^{10}$ Seattle Children's Hospital, Division of Pediatric Otolaryngology, Department of Otolaryngology/Head and Neck Surgery, University of Washington, Seattle, Washington 98105, USA;

${ }^{11}$ Vascular Anomalies Program, Seattle Children's Hospital, Seattle, Washington 98105, USA
\end{abstract}

Corresponding author: jtbenn@uw.edu

(C) 2021 Sheppard et al. This article is distributed under the terms of the Creative Commons Attribution-NonCommercial License, which permits reuse and redistribution, except for commercial purposes, provided that the original author and source are credited.

Ontology terms: frontal venous angioma; hemorrhage of the eye; intracranial hemorrhage;

lymphangioma; ocular pain

Published by Cold Spring Harbor Laboratory Press

doi:10.1101/mcs.a006147
Abstract Disorganized morphogenesis of arteries, veins, capillaries, and lymphatic vessels results in vascular malformations. Most individuals with isolated vascular malformations have postzygotic (mosaic), activating pathogenic variants in a handful of oncogenes within the PI3K-RAS-MAPK pathway (Padia et al., Laryngoscope Investig Otolaryngol 4: 170-173 [2019]). Activating pathogenic variants in the gene PIK3CA, which encodes for the catalytic subunit of phosphatidylinositol 3-kinase, are present in both lymphatic and venous malformations as well as arteriovenous malformations in other complex disorders such as CLOVES syndrome (congenital, lipomatous, overgrowth, vascular malformations, epidermal anevi, scoliosis) (Luks et al., Pediatr Dev Pathol 16: 51 [2013]; Luks et al., J Pediatr 166: 1048-1054.e1-5 [2015]; Al-Olabi et al., J Clin Invest 128: 1496-1508 [2018]). These vascular malformations are part of the PIK3CA-related overgrowth spectrum, a spectrum of entities that have regionalized disordered growth due to the presence of tissue-restricted postzygotic PIK3CA pathogenic variants (Keppler-Noreuil et al., Am J Med Genet A 167A: 287-295 [2015]). Cerebrofacial vascular metameric syndrome (CVMS; also described as cerebrofacial arteriovenous metameric syndrome, BonnetDechaume-Blanc syndrome, and Wyburn-Mason syndrome) is the association of retinal, facial, and cerebral vascular malformations (Bhattacharya et al., Interv Neuroradiol 7: 517 [2001]; Krings et al., Neuroimaging Clin N Am 17: 245-258 [2007]). The segmental distribution, the presence of tissue overgrowth, and the absence of familial recurrence are all consistent with CVMS being caused by a postzygotic mutation, which has been hypothesized by previous authors (Brinjiki et al., Am J Neuroradiol 39: 2103-2107 [2018]). However, the genetic cause of CVMS has not yet been described. Here, we present three individuals with CVMS and mosaic activating pathogenic variants within the gene PIK3CA. We propose that CVMS be recognized as part of the PIK3CA-related overgrowth spectrum, providing justification for future trials using pharmacologic PIK3CA inhibitors (e.g., alpelisib) for these difficult-to-treat patients. 


\section{CASE PRESENTATION}

\section{Participant 1}

Participant 1 is a 4-yr-old female with cerebrofacial vascular metameric syndrome (CVMS) consisting of a right orbital/periorbital microcystic lymphatic malformation, bilateral cerebellar developmental venous anomalies, and venous sinus thrombosis. She was found to have fetal sinus thrombosis on prenatal ultrasound, confirmed by fetal magnetic resonance imaging (MRI) and postnatal MRI. Right periorbital and preseptal soft tissue swelling was also noted on fetal MRI. She was noted to have intraventricular hemorrhage on postnatal imaging and developed forehead asymmetry around 3 mo of age. Ultrasound at 5 mo showed an ill-defined vascular lesion in the right orbit. An MRI of the orbit, face, and neck and magnetic resonance venography of the brain at 6 mo of age demonstrated a T1 hypointense, T2 hyperintense lesion in the right superior periorbital and temporalis region. Extensive venous anomalies including bilateral developmental venous anomalies (DVAs) within the cerebellum, distension of the vein of Galen and superior sagittal sinus, and extensive abnormal venous drainage throughout the right cerebral hemisphere (Fig. 1). Repeat imaging just before $3 \mathrm{yr}$ of age showed interval thrombosis of deep intraorbital venous components, as well as a dominant cystic intraorbital component with mass effect on the globe, and a multiloculated microcystic component of the right supraorbital/brow soft tissues and stable venous anomalies.

Her medical history was significant for speech delay, epistaxis of the right nare requiring cauterization, and amblyopia. There was a family history of speech delay and no family
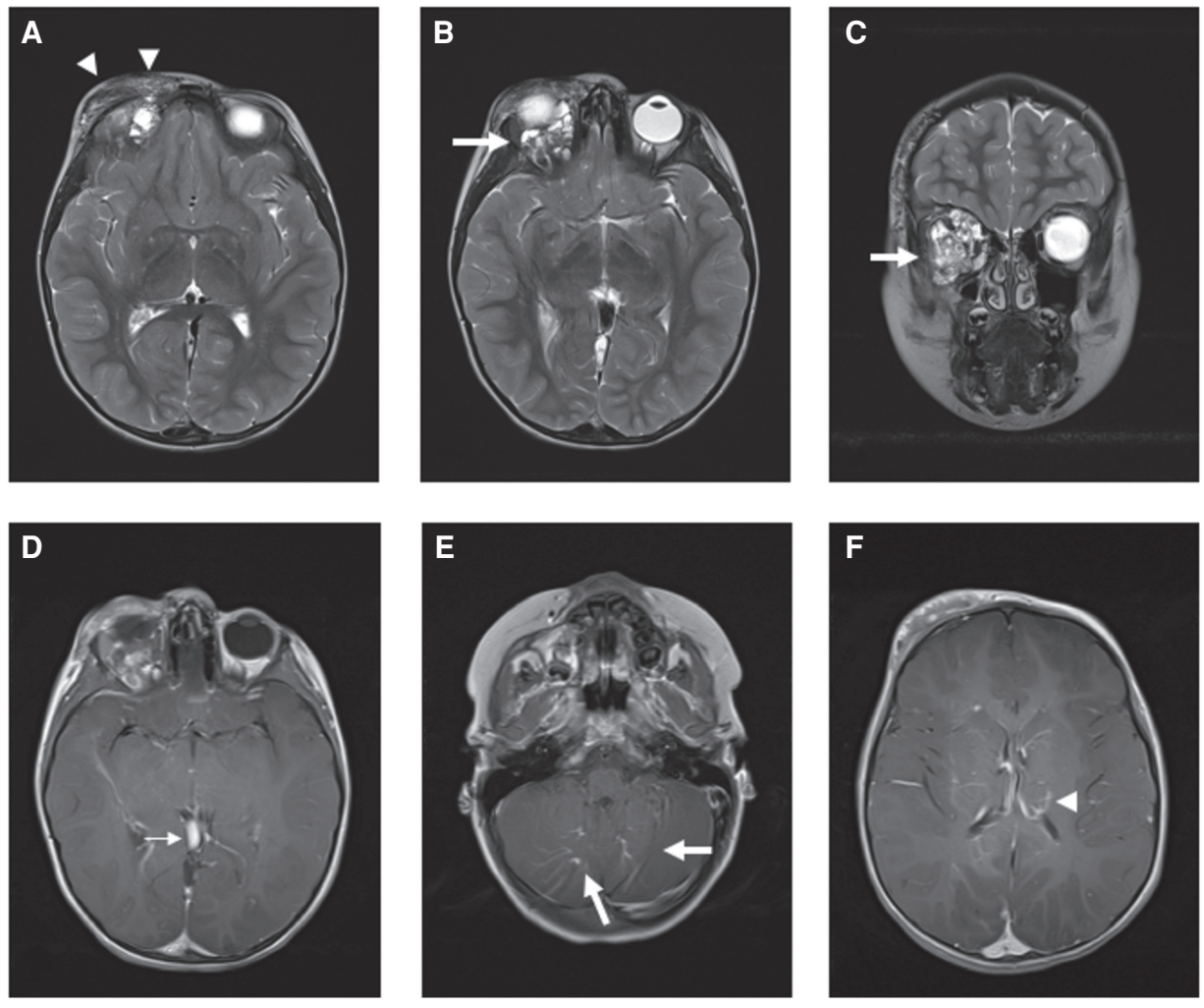

Figure 1. Vascular malformations in Participant 1 from a postcontrast T1-weighed MRI of the brain. (A) Short arrows indicate microcystic periorbital component. $(B, C) A$ confluent microcystic component (albeit with larger cysts) in the retro-orbital and intraconal space. (D) Thin arrow: prominent vein of Galen. $(E)$ Long arrows: cerebellar developmental venous anomalies. $(F)$ Arrowheads: left thalamic developmental venous anomaly. 
Table 1. Mosaic pathogenic variants in PIK3CA

\begin{tabular}{lccllrr}
\hline Gene & Chr & c.DNA (NM_006218) & \multicolumn{1}{c}{ Protein } & Variant type & Predicted effect & Genotype (VAF) \\
\hline PIK3CA & 3 & c.1633G $>$ A & p.Glu545Lys & Substitution & GOF & $3 \%$ \\
PIK3CA & 3 & c.3140A $>$ G & p.His1047Arg & Substitution & GOF & $4.3 \%$ \\
PIK3CA & 3 & c.1624G $>$ A & p.Glu542Lys & Substitution & GOF & $2 \%$ \\
\hline
\end{tabular}

(VAF) Variant allele frequency, (GOF) gain of function.

history of vascular malformations. Her physical exam was unremarkable except for periorbital soft tissue fullness, ptosis, and visible vessels in the right eye.

Prior to $1 \mathrm{yr}$ of age, oral sirolimus was trialed for 3 mo with no improvement on physical exam, although subtherapeutic sirolimus levels were noted on two occasions. She had seven sclerotherapy treatments (1-3.5 $\mathrm{mL}$ of $5 \%$ ethanolamine oleate every 6-8 wk) between $18 \mathrm{mo}$ and $3 \mathrm{yr}$. She was treated with three units of bleomycin sclerotherapy at $4 \mathrm{yr}$ and 8 units at $5 \mathrm{yr}$. Excisional biopsy of her right orbital/periorbital lymphatic malformation showed fibrous tissue in which lie adipose tissue and skeletal muscle with occasional nerves accompanied by small arteries and veins in a normal quantity and distribution with few scattered slit-like thin-walled vascular spaces. The endothelium lining the spaces was positive for D2-40 by immunohistochemical staining.

Sequencing of genomic DNA isolated from frozen malformation tissue using the VANSeq panel (see below) identified a somatic pathogenic variant in PIK3CA (NM 006218:c.1633G > A; p.Glu545Lys; Table 1) at 3\% variant allele frequency (VAF; $n=1523$ reads) that was absent from blood ( $n=1616$ reads).

\section{Participant 2}

Participant 2 is a 19-mo-old female with CVMS consisting of a left-sided facial vascular malformation and an aberrant left-sided intracranial venous anomaly. She presented after birth with swelling of the left eye and forehead. Postnatal ultrasound and MRI demonstrated a predominantly lymphatic malformation centered on the left frontal scalp soft tissues and a predominantly venous component involving the greater sphenoid wing and left orbital roof and lateral wall. Intracranially, there was dilation of the sphenoparietal sinus with venous varix at the left temporal pole and a small venous pouch in the posterior extraconal left orbit, in continuity with the left cavernous sinus. There was no associated malformation of cortical development.

Because of her risk for amblyopia, she was treated with patching. At 9 mo, she was treated with surgical excision of the left temporal lymphatic malformation with local flap reconstruction. Pathology showed multiple dilated, irregular, and anastomosing vascular channels lined by variably thick walls with discontinuous smooth muscle lining consistent with lymphatic channels. Repeat imaging at $1 \mathrm{yr}$ of age showed a decrease in the size of the lesion centered in the left frontal scalp but an increase in the size of the sphenoid/maxillary component, with features suggestive of internal hemorrhage. Eye examination at this time showed increasing proptosis and increased intraocular pressures and was treated with a course of steroids. Repeat MRI at 18 mo showed increased size of orbital component of vascular lesion, with possible stretching of optic nerve, so she was started on sirolimus at $0.8 \mathrm{mg} / \mathrm{m}^{2}$, with goal trough level of $5-10 \mathrm{ng} / \mathrm{mL}$. She has tolerated this dose of sirolimus for 2 mo but not enough time has passed to determine if she is responding.

Droplet digital polymerase chain reaction (ddPCR) identified a somatic pathogenic variant in PIK3CA (NM_006218:c.3140A > G, p.His1047Arg; Table 1) in lymphatic cyst fluid- 
derived cell-free DNA at $4.3 \%$ variant allele frequency $(n=276$ mutant and 6256 wild-type droplets). This variant was not detected in blood.

\section{Participant 3}

Participant 3 is a 22-yr-old male presenting with CVMS consisting of left orbital vascular malformation and left temporal developmental venous anomaly. At birth, his left pupil was noted to be more dilated than the right. MRI at 3 years old showed left orbital multiloculated mass with evidence of hemorrhage as well as developmental venous anomalies within the left temporal, parietal, and occipital lobes. At 6 years old, he underwent partial resection of his orbital vascular malformation, which showed a collection of thin-walled vessels with adipose tissue and skeletal muscle, with scattered aggregates of chronic inflammatory cells and histiocytes. He reported frequent epistaxis with headaches. Because of severe headaches and proptosis, he underwent exenteration of his left eye. Pathology showed a vascular malformation involving external surface of the eye, abutting the sclera but not involving optic nerve or sclera and not penetrating the globe (Fig. 2). Dental examination noted an ectopic left maxillary tooth. Repeat MRI at 14 years old showed postsurgical changes of the left orbital lymphatic malformation and left globe enucleation. The appearance of a developmental venous anomaly and enlarged draining venous structures in the left cerebral hemisphere appeared stable from the prior study. Despite the left globe enucleation, his severe headaches persisted and were treated with narcotic pain medication. At 22 years old, MRI showed a benign-appearing cystic lesion with a fluid level in the anterior aspect of the left temporal lobe compatible with cavernous malformation as well as chronic ectasia of the venous structures on the posterior aspect of the left cerebral hemisphere. This temporal lobe cavernous malformation was located in the same region that previously demonstrated a developmental venous anomaly. Imaging at 23 years old showed increased size of the cavernous malformation within his left temporal lobe, now with evidence of acute intralesional hemorrhage.

Sequencing of genomic DNA isolated from formalin-fixed vascular malformation tissue using the VANSeq panel identified a somatic pathogenic variant in PIK3CA (NM_006218: c.1624G > A, p.Glu542Lys; Table 1 ) at $2 \% \operatorname{VAF}(n=905$ reads). His blood was not tested for this variant.

\section{Technical Analyses}

The VANSeq test (https://seattlechildrenslab.testcatalog.org/show/LAB1920-1 for more information) was used for Participants 1 and 3. Briefly, targeted capture of 44 genes, including PIK3CA, was performed using a custom-designed Integrated DNA Technologies panel. The target region includes all coding exons and at least 10 bp of flanking sequence. Libraries were sequenced on an Illumina NextSeq with 150 bp paired end sequencing. Average coverage across the entire target was 1940 x and 1884 × for Participant 1's tissue and blood, respectively, and $1098 \times$ for Participant 3's tissue. After sequencing, reads were aligned with Novoalign and variants called with FreeBayes, GATK, and Pindel. Participant 2 was tested using a multiplex ddPCR assay that simultaneously detects the four most common PIK3CA variants observed in cancer and lymphatic malformations (p.Glu545Lys, p.Glu542Lys, p. His1047Arg, and p.His1047Leu), as previously described (Zenner et al. 2021).

\section{GENOMIC FINDINGS}

\section{Variant Interpretation}

All three variants have previously been reported to be pathogenic in numerous unrelated individuals with PIK3CA-related segmental overgrowth syndrome. These are the most 

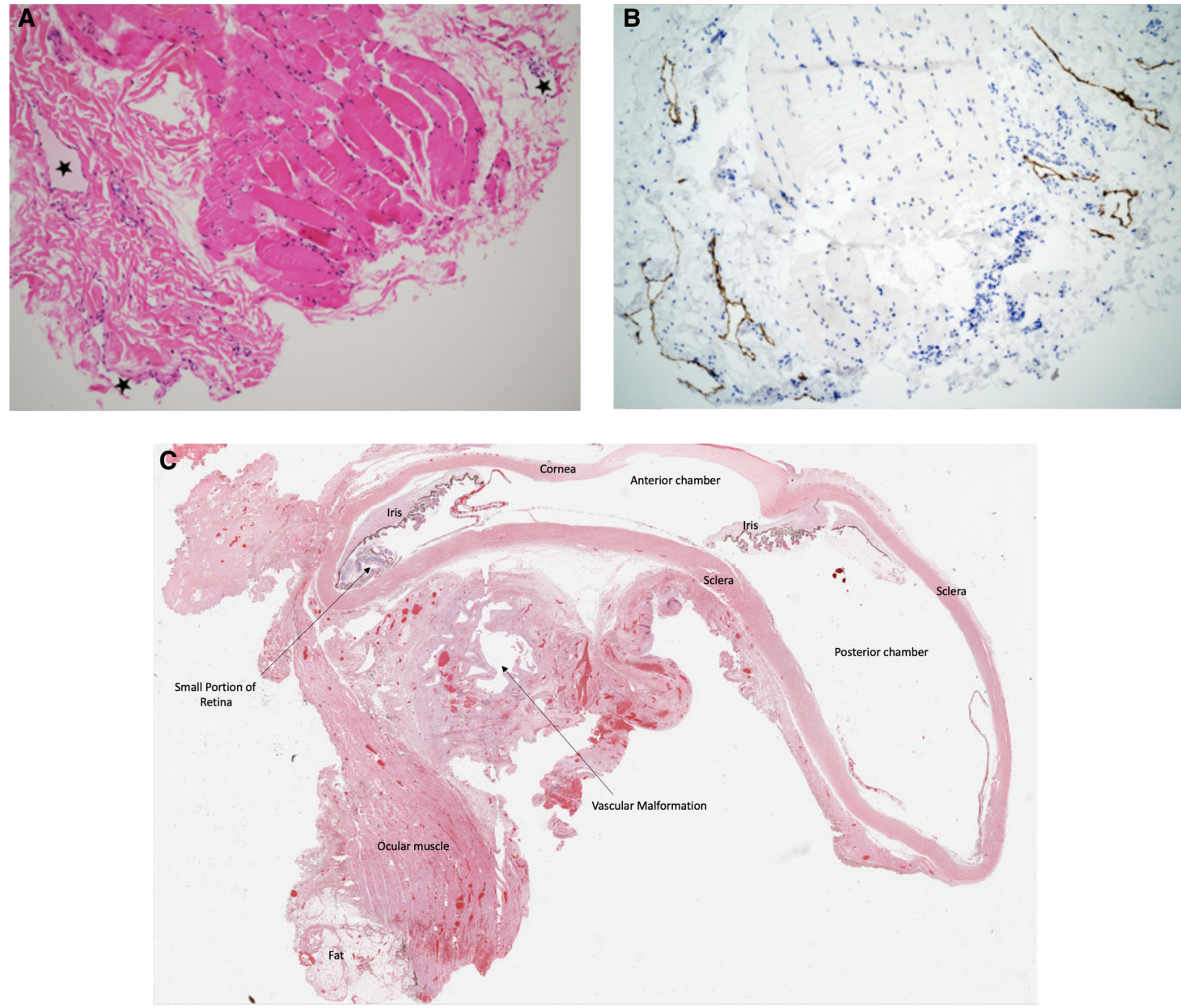

Figure 2. Pathology from Participant 1 and Participant 3. (A) Hematoxylin and eosin staining of the periorbital sample from Participant 1 at $100 \times$ magnification contains fat (not shown), skeletal muscle, and fibrous tissue in which lie scattered mildly dilated thin-walled lymphatic channels (star). (B) The vessels express D2-40, confirming lymphatic differentiation (100x, diaminobenzidine). (C) Vascular malformation from Participant 3 includes dilated channels with flat endothelial lining that are filled with lymphatic fluid and/or blood and focal organizing clot. The surrounding vessel wall includes myxoid tissue with minimal smooth muscle. Normal appearing skeletal muscle is present, adjacent to the vascular lesion. The vascular malformation abuts the sclera but does not penetrate into the globe in sections examined. The structures of the eye, including a partially detached retina, iris, ciliary bodies, and choroid, are not directly involved.

common driver pathogenic variants in PIK3CA-related cancers and are absent from large population databases, and numerous functional studies have demonstrated that they lead to kinase hyperactivation (Dogruluk et al. 2015; Kuentz et al. 2016; Mirzaa et al. 2016; Zenner et al. 2019).

\section{SUMMARY}

We describe three individuals with CVMS (Table 2), characterized by lymphatic malformations involving the orbit and ipsilateral cerebral venous anomalies, all of whom have mosaic activating pathogenic variants in the gene PIK3CA. These findings add to the 


\begin{tabular}{lccc}
\hline Table 2. Clinical features in individuals with cerebrofacial vascular & metameric syndrome (CVMS) & \\
\hline Clinical feature & Participant & Participant & Participant \\
\hline Abnormality of the lymphatic system HP:0100763 & 1 & 2 & 3 \\
Developmental venous anomaly (associated term cerebral & Yes & Yes & Yes \\
venous angioma HP:0012481) & & Yes & Yes \\
Central venous sinus thrombosis HP:0033724 & Yes & No & No \\
Intraventricular hemorrhage HP:0030746 & Yes & No & Yes \\
Spontaneous, recurrent epistaxis HP:0004406 & Yes & No & Yes \\
Amblyopia HP:0000646 & Yes & Yes & No \\
Proptosis HP:0000520 & No & No & Yes \\
Ptosis HP:0000508 & Yes & Yes & No \\
Facial asymmetry HP:0000324 & Yes & Yes & Yes \\
Delayed speech and language development HP:0000750 & Yes & No & No \\
Headache HP:0002315 & No & No & Yes \\
Supernumerary tooth HP:0011069 & No & No & Yes \\
\hline
\end{tabular}

expanding spectrum of clinical presentations associated with PIK3CA and provide justification for future studies examining efficacy of drugs (e.g., sirolimus, alpelisib) in the treatment of CVMS.

The association of retinal, facial, and cerebral vascular malformations was recognized in the 1930s and was originally named after Bonnet-Dechaume-Blanc and Wyburn-Mason, some of the first authors to describe the association. A diagnosis of CVMS requires the presence of a vascular malformation in at least two of three adjacent zones: brain, orbit, and face. These criteria were proposed by Bhattacharya et al. (2001), who was also the first to use the term "metameric syndrome" to describe this condition, reflecting the embryologic origins of the neural crest and adjacent cephalic mesoderm that give rise to vessels of the face and brain. The term metameric syndrome is well-recognized within the radiology literature, and a subclassification system exists, depending on which region is involved. CVMS 1 involves structures derived from the medial prosencephalon (forehead, nose, hypothalamus, and corpus callosum). CVMS 2 involves structures derived from the lateral prosencephalon (maxilla, cheek, orbit, and temporal/parietal/occipital lobes). CVMS 3 involves structures derived from the rhombencephalon/mesencephalon (lower face, mandible, cerebellum, and brainstem) When the vascular malformation is primarily venous, the term CVMS has been used, and when the malformation is primarily arterial, the term cerebrofacial arteriovenous metameric syndrome has been used.

The identification of somatic mosaic pathogenic variants in tissue from individuals with CVMS is not surprising, but has not been previously reported in individuals meeting criteria for CVMS (Bhattacharya et al. 2001; Brinjikji et al. 2018). Our findings are consistent with proposed pathophysiologic mechanism and place CVMS alongside Sturge-Weber syndrome and others as a segmental neurovascular syndrome (Krings et al. 2007).

The presence of ipsilateral deep venous anomalies in individuals with CVMS is consistent with the recent description of somatic gain of function variants in PIK3CA in isolated cerebral cavernous malformations (CCMs), which often develop alongside DVAs (Porter et al. 1997; Abdulrauf et al. 1999; Ren et al. 2021). Participants 1 and 2 in our report had DVAs and may be at increased risk for developing into CCMs later in life. 
Participant 3's DVA ultimately progressed to a CCM that bled in his early 20s. DVAs have previously been associated with facial venous malformations (Brinjikji et al. 2018), suggesting that interval MRI screening of some patients with facial vascular malformations could be considered. Cerebral cavernous malformations associated with PIK3CA mutations are associated with a higher risk of hemorrhage (Hong et al. 2021), supporting future studies examining the efficacy of PI3K-RAS-MAPK pathway inhibitors in reducing CCM progression and bleeding.

\section{METHODS}

Patients were evaluated in multidisciplinary vascular anomaly programs. Sequencing was performed using the VANSeq clinical assay at Seattle Children's Molecular Genetics Laboratory (https://seattlechildrenslab.testcatalog.org/show/LAB1920-1) and multiplex PIK3CA ddPCR was performed as described (Zenner et al. 2021).

\section{ADDITIONAL INFORMATION}

\section{Data Deposition and Access}

The data are not available because of privacy considerations. The variants were submitted to ClinVar (https://www.ncbi.nlm.nih.gov/clinvar/) in association with the CVMS phenotype and under submission numbers SUB10454835, SUB10454826, and SUB10454782.

\section{Ethics Statement}

This study was approved by the Institutional Review Board at Seattle Children's Hospital. Written, informed consent was obtained from each individual involved in this study or their parents, if they were minors.

\section{Acknowledgments}

We thank the participants and their families. We thank the Comprehensive Vascular Anomaly Program at the Children's Hospital of Philadelphia and the Vascular Anomaly Program at Seattle Children's Hospital.

\section{Author Contributions}

S.E.S. and J.T.B. conceived the study and wrote the manuscript. All authors collected data, analyzed the data, and edited and approved the manuscript.

Competing Interest Statement D.A. is an advisor for Novartis, which makes the drug alpelisib mentioned in the article.

Received August 23, 2021; accepted in revised form September 30, 2021.

\section{Funding}

Research reported in the publication was supported by the Children's Hospital of Philadelphia, Uplifting Athletes, the Lymphangiomatosis and Gorhams Disease Alliance (to S.E.S.), R01 HL130996 from the National Heart, Lung, and Blood Institute (to J.T.B.), a Burroughs Wellcome Career Award for Medical Scientists 1014700 (to J.T.B.), and a Seattle Children's Hospital Guild Association Funding Focus Award (to J.P.). The content is solely the responsibility of the authors and does not necessarily represent the official views of these organizations. 


\section{REFERENCES}

Abdulrauf SI, Kaynar MY, Awad IA. 1999. A comparison of the clinical profile of cavernous malformations with and without associated venous malformations. Neurosurgery 44: 41-46. doi:10.1097/00006123199901000-00020

Bhattacharya JJ, Luo CB, Suh DC, Alvarez H, Rodesch G, Lasjaunias P. 2001. Wyburn-Mason or BonnetDechaume-Blanc as cerebrofacial arteriovenous metameric syndromes (CAMS). A new concept and a new classification. Interv Neuroradiol 7: 5-17. doi:10.1177/159101990100700101

Brinjikji W, Hilditch CA, Tsang AC, Nicholson PJ, Krings T, Agid R. 2018. Facial venous malformations are associated with cerebral developmental venous anomalies. Am J Neuroradio/ 39: 2103-2107. doi:10.3174/ ajnr.A5811

Dogruluk T, Tsang YH, Espitia M, Chen F, Chen T, Chong Z, Appadurai V, Dogruluk A, Eterovic AK, Bonnen PE, et al. 2015. Identification of variant-specific functions of PIK3CA by rapid phenotyping of rare mutations. Cancer Res 75: 5341-5354. doi:10.1158/0008-5472.CAN-15-1654

Hong T, Xiao X, Ren J, Cui B, Zong Y, Zou J, Kou Z, Jiang N, Meng G, Zeng G, et al. 2021. Somatic MAP3K3 and PIK3CA mutations in sporadic cerebral and spinal cord cavernous malformations. Brain 144: 2648-2658. doi:10.1093/brain/awab117

Krings T, Geibprasert S, Luo CB, Bhattacharya JJ, Alvarez H, Lasjaunias P. 2007. Segmental neurovascular syndromes in children. Neuroimaging Clin N Am 17: 245-258. doi:10.1016/j.nic.2007.02.006

Kuentz P, Duffourd Y, St-Onge J, Jouan T, Sorlin A, Thauvin-Robinet C, Thevenon J, Rivière J, Faivre L, Vabres P. 2016. Mutational spectrum in PIK3CA-related overgrowth spectrum (PROS) and recommendations for molecular testing. J Invest Dermatol 136: S192. doi:10.1016/j.jid.2016.06.204

Mirzaa G, Timms AE, Conti V, Boyle EA, Girisha KM, Martin B, Kircher M, Olds C, Juusola J, Collins S, et al. 2016. PIK3CA-associated developmental disorders exhibit distinct classes of mutations with variable expression and tissue distribution. JCl Insight 1: 16. doi:10.1172/jci.insight.87623

Porter PJ, Willinsky RA, Harper W, Wallace MC. 1997. Cerebral cavernous malformations: natural history and prognosis after clinical deterioration with or without hemorrhage. J Neurosurg 87: 190-197. doi:10.3171/ jns.1997.87.2.0190

Ren AA, Snellings DA, Su YS, Hong CC, Castro M, Tang AT, Detter MR, Hobson N, Girard R, Romanos S, et al. 2021. PIK3CA and CCM mutations fuel cavernomas through a cancer-like mechanism. Nature 594: 271276. doi:10.1038/s41586-021-03562-8

Zenner K, Cheng CV, Jensen DM, Timms AE, Shivaram G, Bly R, Ganti S, Whitlock KB, Dobyns WB, Perkins J, et al. 2019. Genotype correlates with clinical severity in PIK3CA-associated lymphatic malformations. JCl Insight 4: e129884. doi:10.1172/jci.insight.129884

Zenner K, Jensen DM, Cook TT, Dmyterko V, Bly RA, Ganti S, Mirzaa GM, Dobyns WB, Perkins JA, Bennett JT. 2021. Cell-free DNA as a diagnostic analyte for molecular diagnosis of vascular malformations. Genet Med 23: 123-130. doi:10.1038/s41436-020-00943-8 


\section{COLD SPRING HARBOR Molecular Case Studies}

\section{Cerebrofacial vascular metameric syndrome is caused by somatic pathogenic variants in PIK3CA}

Sarah E. Sheppard, Victoria R. Sanders, Abhay Srinivasan, et al.

Cold Spring Harb Mol Case Stud 2021, 7: a006147

Access the most recent version at doi: $10.1101 / \mathrm{mcs} . a 006147$

References

License

Email Alerting Service
This article cites 12 articles, 2 of which can be accessed free at: http://molecularcasestudies.cshlp.org/content/7/6/a006147.full.html\#ref-list-1

This article is distributed under the terms of the Creative Commons

Attribution-NonCommercial License, which permits reuse and redistribution, except for commercial purposes, provided that the original author and source are credited.

Receive free email alerts when new articles cite this article - sign up in the box at the top right corner of the article or click here. 